\title{
Effect of temperature and moisture on the uniformity of delivered dose, emitted dose and fine particle fraction for three different DPI formulations in different packs
}

\begin{abstract}
The development of a stable dry powder inhalation (DPI) product with the delivery of consistent fine particle fraction until the product expires is challenging for many drug companies. The main purpose of this study was to investigate the influence of both temperature and humidity on the uniformity of delivered dose, emitted dose and fine particle fraction on three different DPI products in two different packs viz. HDPE container and Alu Alu blister during the stability studies. The study has been performed on Budesonide $400 \mathrm{mcg}$, Formoterol fumarate dihydrate $12 \mathrm{mcg}$ and Tiotropium bromide $18 \mathrm{mcg}$ DPI products. The uniformity of delivered dose (UDD) is calculated by using the Dosage Unit Sampling Apparatus (DUSA) and the emitted dose (ED) and fine particle fraction (FPF) are calculated by using the Next Generation Impactor (NGI). All three products have shown no significant reductions in the UDD, Formoterol fumarate showed a significant reduction in ED value and all of them showed a significant reduction in the FPF values. The reduction in FPF was more in Alu Alu blisters as compared to HDPE containers. The outcome of the study could be extrapolated to other DPI formulations.
\end{abstract}

Volume 7 Issue 4 - 2020

\author{
Priyanka Gondhale, Shrikant Kulkarni, \\ Bhaskar Musmade, Pramod Kolsure, \\ Shrikant Dhavale, Shrinivas Bhope, Sriram \\ Padmanabhan \\ Sava Healthcare Limited, Research Centre, India
}

Correspondence: Shrinivas Bhope, Sava Healthcare Limited, Research Centre, Block DI, Plot no. 17/6, MIDC, Chinchwad, Pune-4II0I9, India, Email shrinivas.hope@savaglobal.com

Received: July 30, 2020 | Published: August 20, 2020

Keywords: fine particle fraction, emitted dose, stability study, nextgen impactor, dosage unit sampling apparatus

Abbreviations: DPI, dry powder inhalation; HDPE, highdensity polyethylene; UDD, uniformity of delivered dose; DUSA, dosage unit sampling apparatus; ED, emitted dose; FPF, fine particle fraction; NGI, next generation impactor; COPD, chronic obstructive pulmonary disease; APSD, aerodynamic particle size distribution; ICH, International conference on harmonization; HPLC, high performance liquid chromatography; MP, mouth piece adaptor; IP, Induction Port; LOD-limit of detection; LOQ, limit of quantitation; API, active pharmaceutical ingredient; CEO, chief executive officer

\section{Introduction}

The inhalation therapy for the treatment of asthma and chronic obstructive pulmonary disease (COPD) has been in use for many years. The drug is directly made available in the lung region in inhalation formulations in comparison with oral or parenteral formulations. Due to this, the unwanted systemic effects of the drugs are minimized with a rapid onset of action. Hence, the dry powder inhalation formulations of bronchodilator and corticosteroid class are commonly prescribed for asthma and COPD. ${ }^{1,2}$ In DPI formulations, many factors potentially influence the aerodynamic performance of the drugs. The polymorphism, crystal habit of drug substance, drug to carrier ratio, the particle size distribution of the drug and the carrier, storage conditions, the pack resistivity for both temperature and humidity would influence the regional deposition of the drug in the lungs and further to the drug's clinical efficacy. ${ }^{3}$ The moisture makes a significant impact on the aerosolization of the drug powder after inhalation by a patient. This ultimately leads to the lowering of fine particle mass. The in vitro particle size distribution of inhalation products is performed by determining the aerodynamic particle size distribution (APSD) by using various cascade impactors. This technique separates the inhaled dose into a series of fractions based on their aerodynamic diameter. The fraction with a particle size range of
1-5 $\mu \mathrm{m}$ gets deposited in the lung region. This fraction is termed as the fine particle fraction (FPF). ${ }^{4}$ For the present study, we have selected Budesonide, Formoterol, and Tiotropium molecules very commonly prescribed for chronic obstructive pulmonary disease (COPD). All the three products are widely available in the market from different pharmaceutical companies as a conventional single dose dry powder inhaler (DPI) products either in Alu-Alu or HDPE pack for the treatment of asthma. ${ }^{5,6}$ The FPF of the emitted dose with the particle size of $1-5 u^{7}$ is a result of so many complex factors ${ }^{8}$ which primarily include inhaler design and device resistance, inspiratory flow by the patient, a particle size of both the API and carrier molecule. ${ }^{9-15}$ Most inhalers deliver FPFs between $20 \%$ and $30 \%$ of the label claim at pressure drops between 2 and $4 \mathrm{kPa}$. Earlier, Xinghan et al., ${ }^{16}$ have studied the effect of temperature and humidity on the nebulizer solution dosage form by using the laser diffraction technique, Ammari et al., ${ }^{17}$ have studied the impact of patients' real-life environmental moisture and temperature conditions on the FPF of Tiotropium DPI. This study is mostly based on the real-time environmental exposure of the inhaler device at patients' end during its application.In this article for the first time, the effect of both the temperature and moisture is studied on three different products in two different packs subjected to 6 months accelerated (40/75) and real-time (25/60) stability as per ICH guidelines. ${ }^{18}$ This study will help to understand the impact of both temperature and humidity on all three important product specifications (UDD, ED, and FPF) and accordingly designing primary pack during the development of capsule-based inhalation dosage form.

\section{Materials and methods}

\section{Reagents and materials}

The Budesonide, Formoterol, and Tiotropium DPI formulations were provided by Sava Healthcare Limited, (Surendranagar, Gujrat, 
India). The RS01 dry powder inhaler model 7 (Plastiape, S.p.a., Italy), was used as a device for all three products to maintain a similar air flow rate.The HPLC grade Acetonitrile, Methanol, and Water (Ranchem, India) were used for the preparation of diluents and mobile phases of all the three products. The analytical grade sodium dihydrogen phosphate monohydrate and orthophosphoric acid (Fisher Scientific, USA) were used as buffers. All other chemicals and reagents were of analytical grade unless otherwise mentioned. The HPLC method details are mentioned in Table 1.

Table I HPLC method parameters

\begin{tabular}{|c|c|c|c|}
\hline Method parameters & Budesonide & Formoterol fumarate & Tiotropium bromide \\
\hline Pump mode & Isocratic & Isocratic & Isocratic \\
\hline Diluent & Buffer: Methanol (30:70) & Water :Methanol (50:50) & Buffer: Methanol (30:70) \\
\hline Buffer & Sodium dihydrogen phosphate $\mathrm{pH} 3.2$ & 0.1\% Ortho phosphoric acid pH 2.I & Sodium dihydrogen phosphate $\mathrm{pH} 3.0$ \\
\hline Mobile phase & Buffer:Acetonitrile (65:35) & Buffer:Acetonitrile : Methanol(70:15:15) & Buffer:Acetonitrile (70:30) \\
\hline HPLC column & Hypersil BDS C8,4.6*250,5 $\mu$ & Hypersil BDS C8,4.6*250,5 $\mu$ & Inertsil ODS 3V, CI8,4.6*250,5 $\mu$ \\
\hline Wavelength & $245 \mathrm{~nm}$ & $215 \mathrm{~nm}$ & $238 \mathrm{~nm}$ \\
\hline Flow rate & $\mathrm{I} .5 \mu \mathrm{L}$ & $1.0 \mu \mathrm{L}$ & $0.7 \mu \mathrm{L}$ \\
\hline Injection volume & $20 \mu \mathrm{L}$ & $100 \mu \mathrm{L}$ & $50 \mu \mathrm{L}$ \\
\hline Run time & 25 Min & $20 \mathrm{Min}$ & 30 Min \\
\hline Retention time & Epimer B-15 Min, Epimer A-16 Min & 10 Min & II Min \\
\hline
\end{tabular}

\section{Formulation development}

All three formulations were developed in house by using respiratory grade APIs and excipients. Respitose SV003, Pharmatose $450 \mathrm{M}$, Inhalac 400, and Inhalac 251 grade excipients in different compositions were used during the development of the formulation.

\section{Instrumentation}

In vitro powder deposition was tested using the NGI and DUSA (Copley Scientific, Nottingham, UK) apparatus. The fractions were analyzed by using the LC $2010 \mathrm{C}_{\mathrm{HT}}$ system (Shimadzu Corporation, Kyoto, Japan). The HPLC instrument consisted of a binary pump, an online degasser, an auto-sampler, a thermostatically controlled column compartment, and a Photo diode array detector (SPD-MZOA). The data were collected and processed using Lab solution ${ }^{\mathrm{TM}}$ software from Shimadzu. RP HPLC method was used for the quantification of all NGI fractions and UDD samples. The stability study as per the ICH guidelines (Q1R2) was carried out by using the stability chambers (Thermolab, India) maintained at $25^{\circ} \mathrm{C} / 60 \% \mathrm{RH}$ and $40^{\circ} \mathrm{C} / 75 \% \mathrm{RH}$ up to 6 Months.

\section{In vitro study}

The total delivered dose for 10 individual capsules for all stability samples was determined using Dosage Unit Sampling Apparatus (DUSA) for the determination of UDD. The emitted dose for every individual capsule was recovered using the respective products diluent. The glass fiber filter (Make: Pall Corporation, New York, USA) used was immersed into the diluent solution and sonicated for $20 \mathrm{~min}$ to dissolve the substance retained on the filter. After every dose emission, the device and capsule shell was thoroughly washed to calculate the residual amount left. The validated HPLC methods were used for all three products. The average delivered dose of all the three products was further used in the calculation of FPF by using the NGI.In vitro powder deposition for all the stability samples was tested using the NGI. Before use, all stages were coated with $1 \%$ of Polypropylene glycol in iso-octane to minimize powder bouncing. A size 3 gelatin capsule with the fill weight of $25 \mathrm{mg}$ for Budesonide and Formoterol Fumarate and $15 \mathrm{mg}$ for Tiotropium bromide mixed with inhalation grade lactose was placed into the sample compartment of a Plastiapemonodose inhaler, the inhaler was activated, inserted into a United States Pharmacopoeia (USP) throat. The flow rate was kept at $69 \mathrm{~L}$ per min to generate a pressure drop of $4 \mathrm{kPa}$ in the device by using the empty capsule based on the USP recommendation ${ }^{19}$ for the in vitro characterization. Accordingly, the test flow duration was set at $3.5 \mathrm{~s}$ by the critical flow controller to draw a $4 \mathrm{~L}$ simulated inhalation volume from the device. After the run, the capsule, inhaler, adaptor mouthpiece, throat, and NGI stages were washed with $50 \mathrm{~mL}$ of diluent which was assayed for solute concentration by HPLC.

Data analysis: The Copley Inhaler Testing Data Analysis Software (CITDAS version 2.0, Copley Scientific Ltd., UK) was used to calculate the FPF.

HPLC quantification: After each determination, the mouthpiece adaptor (MP), induction port (IP), NGI collecting plates, capsules and device were separately rinsed with various volumes of the diluent: MP $(15 \mathrm{~mL}), \mathrm{IP}(15 \mathrm{~mL})$, pre-separator $(50 \mathrm{~mL})$, collecting cups and device $(15 \mathrm{~mL}$ each) of the diluent solution. The validated HPLC method as per ICH guidelines ${ }^{20}$ was used for the quantitation of each compound. The limit of detection (LOD), the limit of quantification (LOQ), and coefficient of determination $\left(\mathrm{r}^{2}\right)$ values for all the three products are reported in Table 2. During the NGI study, the total emitted dose (ED) was calculated from the cumulative amount of drug deposited in the induction port (USP throat), the pre-separator, and all the stages of the NGI. The fine particle dose (FPD) was the mass associated with particles $<5 \mu \mathrm{m}$. 
Table 2 Validation parameters

\begin{tabular}{llll}
\hline validation parameters & Budesonide & Formoterol fumarate & Tiotropium bromide \\
\hline LOD & $1.30 \mu \mathrm{g} / \mathrm{mL}$ & 0.03 & 0.04 \\
LOQ & $4.11 \mu \mathrm{g} / \mathrm{mL}$ & 0.10 & 0.12 \\
$r^{2}$ & 0.9999 & 0.9997 & 1.0000 \\
\hline
\end{tabular}

\section{Result and discussion}

All three products behaved differently in the ED, UDD, and FPF at the end of 6 months of stability study in both the packs. The stability data for UDD, ED, and FPD for Budesonide, Formoterol fumarate, and Tiotropium bromide is reported in Graphs 1-3 respectively. The significant reduction in UDD and ED (except Formoterol fumarate) was not observed for all the three products. The FPF values for Tiotropium bromide (D90 <2.48) and Formoterol fumarate (D90
<3.27) formulation were found to be significantly higher than Budesonide (D90 <3.39), may be because of smaller API particle size. The particle size data for all the APIs and excipients used are reported in Table 3. The reduction in FPF fraction was more prominent in the Alu Alu pack as compared to the HDPE containet. To further investigate the effect of particle size of excipients in Budesonide formulation, we have formulated the product by changing the proportion of two different grades (fine grade and coarse grade) of lactose (Table 3 ).

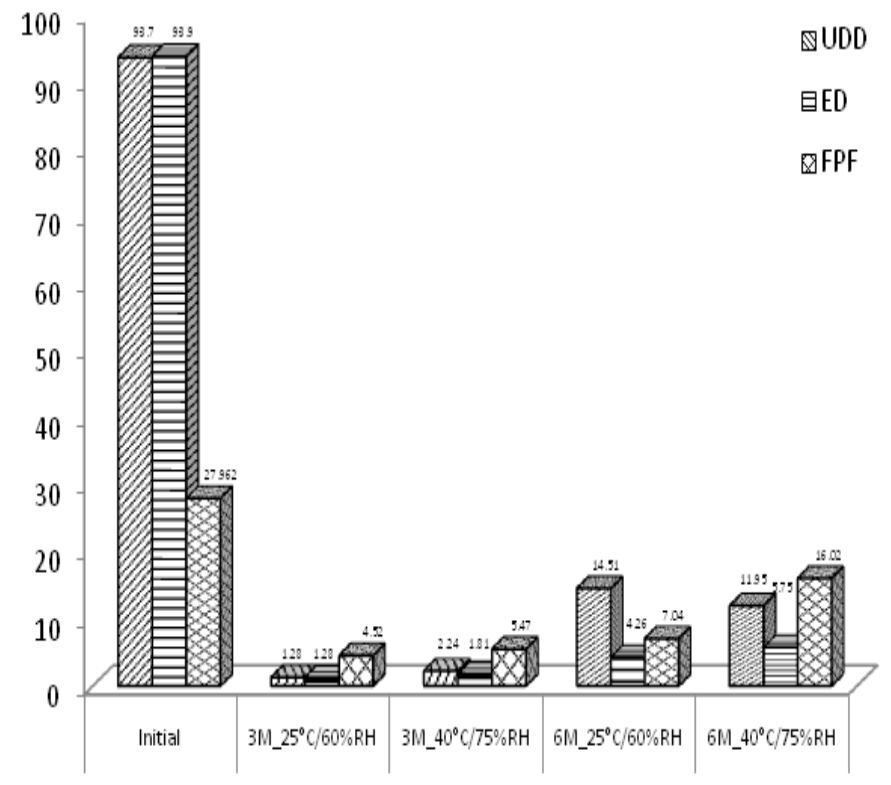

Graph I Budesonide DPI 400mcg (HDPE pack).

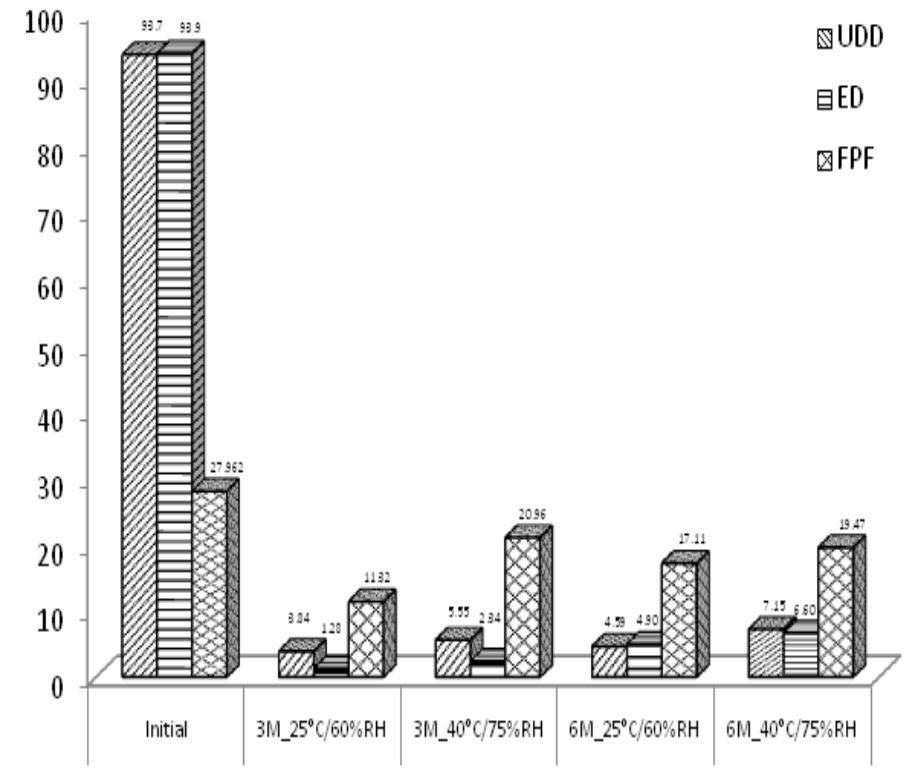

Graph 2 Budesonide DPI 400mcg (ALU-ALU pack).

Citation: Gondhale P, Kulkarni S, Musmade B, et al. Effect of temperature and moisture on the uniformity of delivered dose, emitted dose and fine particle fraction for three different DPI formulations in different packs. J Appl Biotechnol Bioeng. 2020;7(4):I6I-I67. DOI: I0.15406/jabb.2020.07.00229 


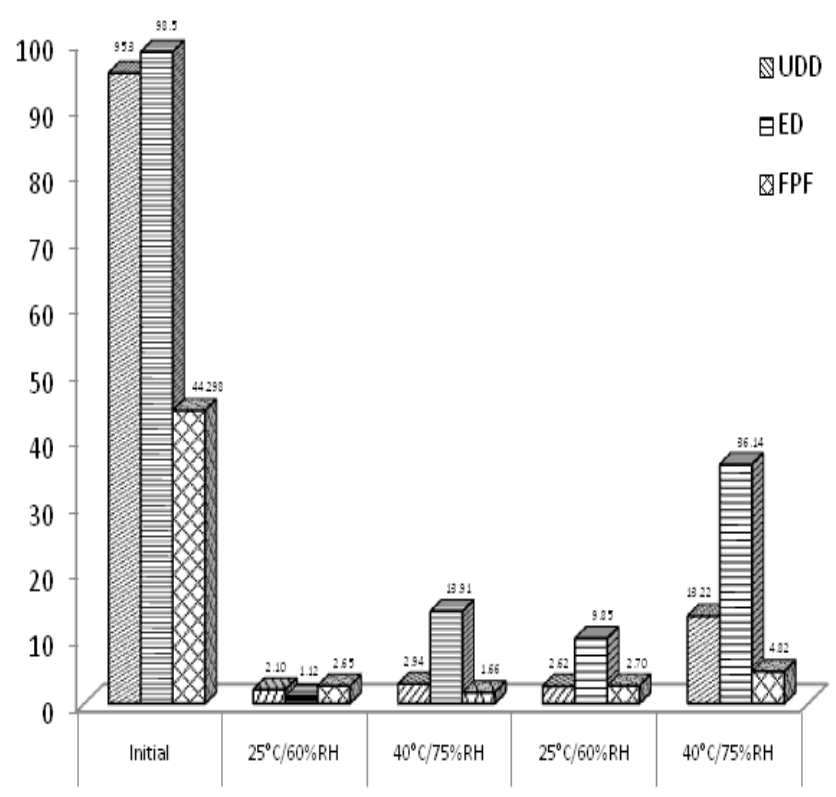

Graph 3 Formoterol Fumarate I2mcg DPI (HDPE pack).

Table 3 Particle size data for the APl's and excipients used

\begin{tabular}{llll}
\hline API/Excipient used & D I0 & D50 & D90 \\
\hline Budesonide & 0.68 & I.5I & 3.39 \\
Formoterol Fumarate & 0.84 & 1.66 & 3.27 \\
Tiotropium bromide & 0.47 & 1.03 & 2.48 \\
Inhalac 25I & 13 & 49 & 89 \\
Respitose SV003 & 31 & 60 & 93 \\
Pharmatose 450 & 3 & 20 & 50 \\
Inhalac 400 & 1.2 & 7.4 & 25.8 \\
\hline
\end{tabular}

In formulation - I, Respitose SV003 and Pharmatose 450 have been used in a ratio of 10:90 and formulation-II in a ratio of 50:50. No significant differences in ED and UDD but significant reduction in FPF $(25.10 \%)$ was seen in formulation-I (Table 4$)$ at the end of 3 months at 40/75\% RH in HDPE pack from the initial value (33.29\%). Interestingly, no further reduction in FPF was observed until 6 months $(25.71 \%)$. There was a very minimal impact on the UDD and ED values in both the batches. In the formulation-II, no significant reduction was observed on UDD, ED, and FPF. After the observation of the significant effect of moisture on the FPF values, studies on the effect of the use of dehydrating reagent (silica gel) packed in the HDPE container was carried out. Surprisingly, because of the strong dehydration by silica, a static charge was generated in the HDPE pack with silica bags. It resulted in the sticking of powder blend inside the HPMC capsules. This resulted in a significant reduction in values for all UDD, ED, and FPF. The data is reported in Table 5.

Table 4 Formulation study with different excipient grades for Budesonide DPI

\begin{tabular}{|c|c|c|c|c|c|c|}
\hline \multirow[t]{2}{*}{ Product Name } & \multirow[t]{2}{*}{ Tests } & \multicolumn{5}{|c|}{ Stability study time points } \\
\hline & & & $3 \mathrm{M} \_25^{\circ} \mathrm{C} / 60 \% \mathrm{RH}$ & $3 \mathrm{M} \_40^{\circ} \mathrm{C} / 75 \% \mathrm{RH}$ & $6 \mathrm{M} \_25^{\circ} \mathrm{C} / 60 \% \mathrm{RH}$ & $6 \mathrm{M} \_40^{\circ} \mathrm{C} / 75 \% \mathrm{RH}$ \\
\hline \multirow[t]{3}{*}{ Formulation-I } & UDD & 89 & 87.8 & 88.1 & 88 & 89.4 \\
\hline & ED & 91.4 & 88.4 & 91.6 & 89.7 & 89.7 \\
\hline & FPF & 33.2 & 23.2 & 25.7 & 24 & 25.7 \\
\hline \multirow[t]{3}{*}{ Formulation-II } & UDD & 87.3 & 87 & 88.5 & 80 & 82.5 \\
\hline & ED & 94.5 & 92.7 & 92.2 & 89.9 & 88.5 \\
\hline & FPF & 26 & 26.4 & 22.1 & 23.4 & 25.9 \\
\hline
\end{tabular}


Table 5 Formulation study with the use of Silica gel for Tiotropium bromide DPI

\begin{tabular}{|c|c|c|c|c|}
\hline \multirow[t]{3}{*}{ Formulation A with dehydrating agent } & UDD & 93.2 & 76.5 & 73.1 \\
\hline & ED & 94.6 & 78.1 & 69.8 \\
\hline & FPF & 38.9 & 31.4 & 29.5 \\
\hline Formulation A without dehydrating agent & FPF & 38.9 & 35.7 & 35.1 \\
\hline
\end{tabular}

No significant reduction in the UDD, ED values were observed but the FPF was significantly affected for all the three products in both the packs. The reduction in ED and FPF is more significant in Alu Alu as compared to the HDPE pack (Graphs 3-6). The FPF for Tiotropium bromide and Formoterol fumarate was found to be significantly higher than Budesonide DPI in the initial analysis. At the end of 6 months stability, the reduction in the FPF for Tiotropium bromide in
Alu pack was highly significant (53.40\%) as compared to Formoterol fumarate dihydrate (18.58\%) may be because of the inbound moisture of Formoterol fumarate molecule in the form of dihydrate, there was no further uptake of moisture during the stability study. The ED value is significantly reduced in Formoterol after 6 months (37.16\%) as compared to Tiotropium bromide (19.78\%) in Alu Alu pack.

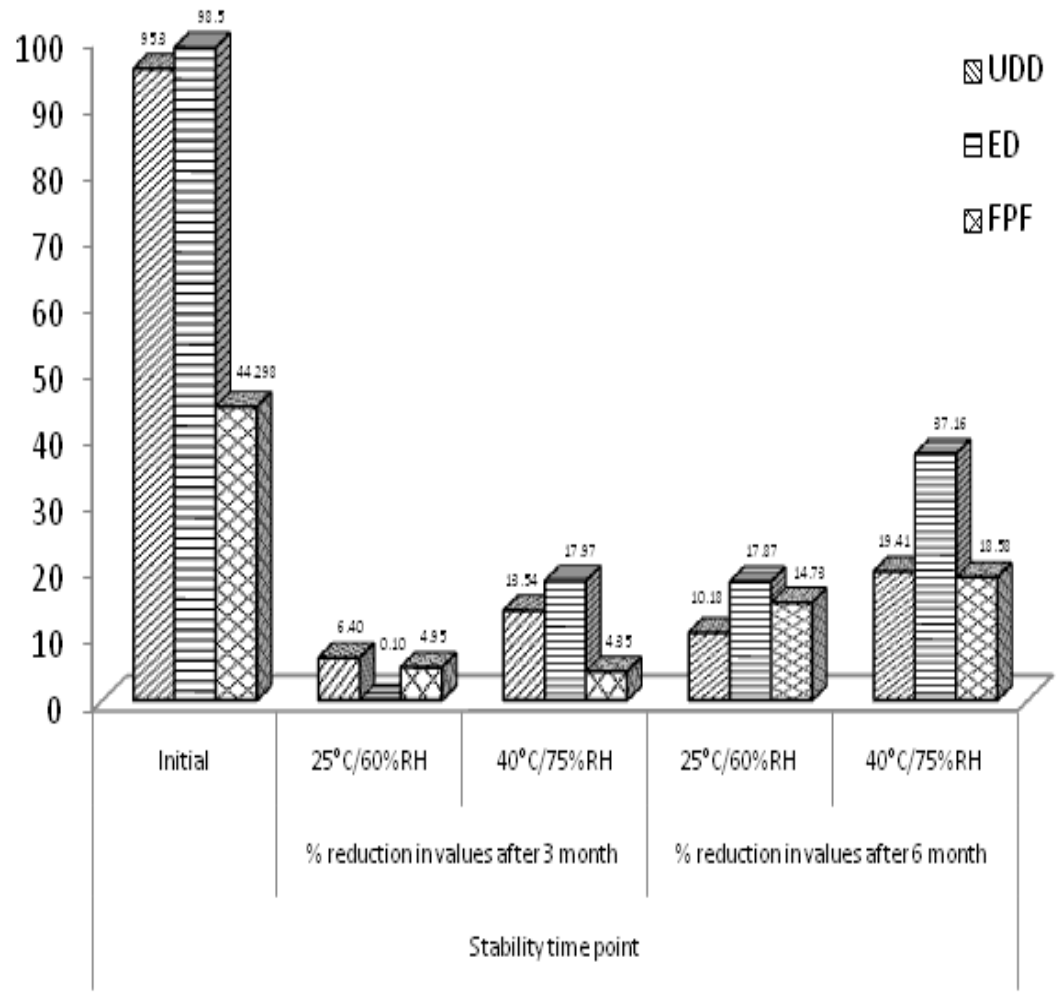

Graph 4 Formoterol Fumarate $12 \mathrm{mcg}$ DPI (ALU-ALU pack). 


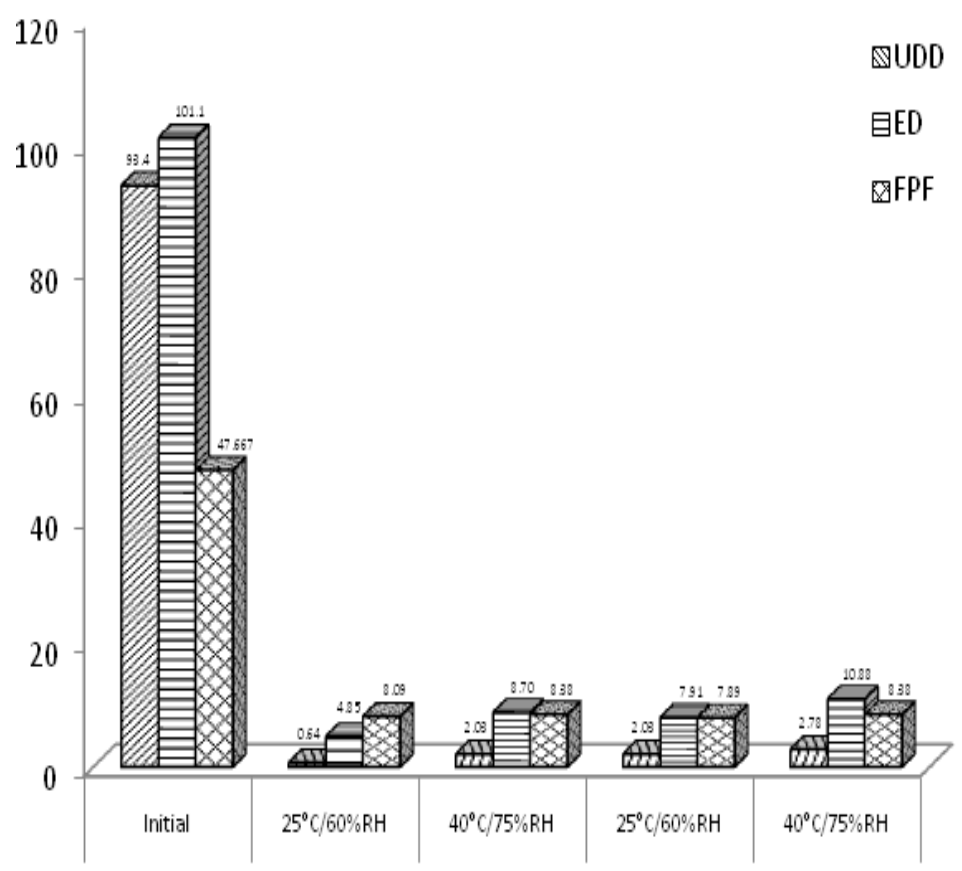

Graph 5 Tiotropium DPI I8mcg (HDPE pack).

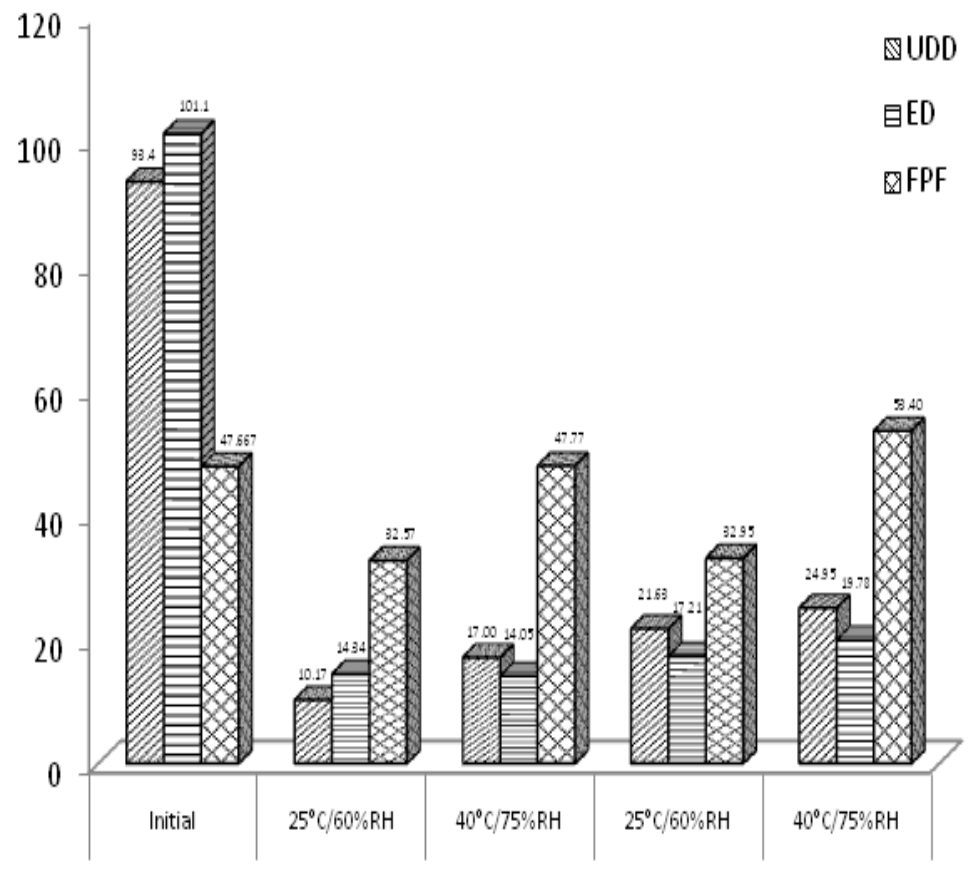

Graph 6 Tiotropium DPI I8mcg (ALU-ALU pack).

\section{Conclusion}

Temperature and humidity play a crucial role in the fine particle fraction; although it has not impacted much to the emitted dose and uniformity of delivered dose. Apart from temperature and moisture; the particle size of the selected excipient also contributes significantly to the determination of FPF. In the present study, it is observed that the use of finer grade API and excipient is leading to the higher FPF at the initial time point. A similar observation has been reported for salbutamol sulphate by Jones etal., ${ }^{21} \&$ Zeng et.al. ${ }^{22}$ This may be because of the increase in the aerosolization of drugs due to a reduction in the mean particle size leading to the enhanced fluidization energy thereby increasing the drug deposition in the lungs in the form of fine particle fraction. ${ }^{23}$ But, during this study, it was observed that with high temperature and moisture, there was a greater risk of moisture absorption by the finer grade API and excipients. It was also noted that the reduction in FPF and ED is not proportionate and varied from product to product. Also, the use of dehydrating reagents like silica bags although reduces the moisture content of the capsule, at the same time, it generates the static charge resulting in the sticking of 
the powder blend to the capsule shell. Hence, the judicial combination of API particle size, the excipient particle size, the validated blending process, and most importantly, the selection of the right pack will ensure the consistency in the FPF throughout the shelf life for any DPI product.

\section{Acknowledgement}

The authors wish to thank Mr. Vinod Ramchandra Jadhav Chairman and Mr. Avinaash Mandale CEO Sava Healthcare Limited for their constant support and encouragement. Thanks are also due to Mr. Sachin Margaj for formatting the figures required for this article.

\section{Conflicts of interest}

The author declares that there is no conflict of interest.

\section{Funding}

None.

\section{References}

1. Global Initiative for Asthma. Global Strategy for Asthma Managemen and Prevention. 2016

2. Celli BR, McNee W, ATS/ERS Task Force. Standards for the diagnosis and treatment of patients with COPD: a summary of the ATS/ERS position paper. Eur Respir J. 2004;23(6):932-46.

3. Chougule MB, Padhi BK, Jinturkar KA, et al, Development of Dry powder Inhalers. Recent Pat Drug Deliv Formul. 2007;1(1):11-21.

4. Laube BL, Janssen HM, de Jong FHC, et al. ERS/ISAM Task force report. What the pulmonologist should know about new inhalational therapies. Eur Respir J. 2011;37:1308-1331.

5. Morice H, Peterson S, Beckman O, et al. Therapeutic comparison of a new budesonide/formoterol pMDI with budesonide pMDI and budesonide/ formoterol DPI in asthma. International Journal of Clinical Practice. 2007;61:1874-1883.

6. Lahelm S, Kirjavainen M, Kela M. "Equivalent lung" deposition of budesonide in vivo: a comparison of dry powder inhalers using a pharmacokinetic method. British Journal of Clinical Pharmacology. 2005;59(2):167-173.

7. Hickey AJ, Martonen TB, Yang Y. Theoretical relationship of lung deposition to the fine particle fraction of inhalation aerosols. Pharm Acta Helv. 1996;71:185-190.

8. Frijlink HW, De Boer AH. Dry powder inhalers for pulmonary drug delivery. Expert Opin Drug Deliv. 2004;1:67-86.
9. Saim S, Horhota ST. Process for overcoming drug retention in hard gelatin inhalation capsules. Drug Dev Ind Pharm. 2002; 28:641-54.

10. Selroos O, Borgstrom L, Ingelf J. Performance of Turbuhaler (R) in patients with acute airway obstruction and COPD, and in children with asthma: understanding the clinical importance of adequate peak inspiratory flow, high lung deposition, and low in vivo dose variability. Treat Respir Med. 2006;5:305-315.

11. Chrystyn H, Price D. Not all asthma inhalers are the same: factors to consider when prescribing an inhaler. Prim Care Respir J e J Gen Pract Airw Group. 2009;18:243-249.

12. de Boer A, Hagedoorn P, Gjaltema D, et al. Air classifier technology (ACT) in dry powder inhalation part 4. Performance of air classifier technology in the Novolizer multi-dose dry powder inhaler. Int J Pharm. 2006;310:81-89.

13. Borgstrom L. On the use of dry powder inhalers in situations perceived as constrained. J Aerosol Med. 2001;14(3):281-287.

14. Agertoft L, Pedersen S. Lung deposition and systemic availability of fluticasone Diskus and budesonide Turbuhaler in children. Am J Respir Crit Care Med. 2003;168:779-782.

15. Newman SP. Aerosol deposition considerations in inhalation therapy. Chest. 1985;88:152-160.

16. Xinghan S, Junhua H, Shuyao Z, et al. Effects of Temperature and Humidity on Laser Diffraction Measurements to Jet Nebulizer and Comparison with NGI. AAPS PharmSciTech. 2016;17(2):380-388.

17. Wesam GA, Mohammad K, Rabab F. The impact of patients' real-life environmental temperature and humidity use conditions of tiotropium dry powder inhaler on its aerosol emission characteristics. European Journal of Pharmaceutical Sciences. 2019;133:137-144.

18. ICH. Q1 (R2): Stability testing of new drug substances and products, International Conference on Harmonization, Geneva; 2003.

19. United States Pharmacopeia, USP 38-NF 33. 2014

20. ICH. Q2 (R1): Validation of Analytical Procedures: Text and Methodology, International Conference on Harmonization, Geneva; 2005.

21. Jones MD, Price R. The influence of fine excipient particles on the performance of carrier-based Dry Powder Inhalation Formulations. Pharm Res. 2006;23(8):1665-1674.

22. Xeng XM, Martin G P, Tee SK, et al. The role of fine particle lactose on the dispersion and deaggregation of salbutamol sulphate in an air stream in vitro. Int J Pharm. 1998;176:99-110.

23. Shur J, Haris H, Jones M, et al. The role of fines in the modification of the fluidization and dispersion mechanism within Dry Powder Inhaler formulations. Pharmaceutical Research. 2008;25(7):1631-1640. 